\title{
Infectious diseases, autoimmunity and midline defect in a patient with a novel bi-allelic mutation in IL12RB1 gene
}

\begin{abstract}
Bahar Göktürk ${ }^{1}$, İsmail Reisli², Ümran Çalışkan ${ }^{3}$, Carmen Oleaga-Quintas ${ }^{4,5}$, Caroline Deswarte ${ }^{4,5}$, Tuba Turul-Özgür ${ }^{6}$, Durmuş Burgucu ${ }^{7}$, Mélanie Migaud ${ }^{4,5}$, Jean-Laurent Casanova $4,5,8,9,10$, Capucine Picard 4,5,8, 9,11, Jacinta Bustamante ${ }^{4,5,9,11}$

${ }^{1}$ Division of Pediatric Allergy and Immunology, Department of Pediatrics, Baskent University Faculty of Medicine, ${ }^{2}$ Division of Pediatric Allergy and Immunology, Department of Pediatrics, and ${ }^{3}$ Division of Pediatric Hematology, Necmettin Erbakan University Meram Faculty of Medicine, Konya, Turkey, ${ }^{4}$ Laboratory of Human Genetics of Infectious Diseases, Necker Branch, Institut National de la Santé et de la Recherche Médicale, INSERM-U1163, 5 Paris Descartes University, Imagine Institute, Paris, France, ${ }^{6}$ Division of Pediatric Allergy and Immunology, Department of Pediatrics and ${ }^{7}$ Antalya Technopark Babylife Cord Blood Bank and Stem Cell Research Center, Antalya, Turkey, ${ }^{8}$ Pediatric Hematology-Immunology Unit, Necker Hospital for Sick Children, Paris, France, ${ }^{9}$ St. Giles Laboratory of Human Genetics of Infectious Diseases, Rockefeller Branch, The Rockefeller University, New York, USA, ${ }^{10}$ Howard Hughes Medical Institute, New York, USA, ${ }^{11}$ Center for the Study of Primary Immunodeficiencies, Assistance Publique-Hôpitaux de Paris AP-HP, Necker-Enfants Malades Hospital, Paris, France. E-mail: gokturkbahar@yahoo.com
\end{abstract}

Received: 14 July 2015, Revised: 16 September 2015, Accepted: 7 October 2015

SUMMARY: Göktürk B, Reisli İ, Çalışkan Ü, Oleaga-Quintas C, Deswarte C, Turul-Özgür T, Burgucu D, Migaud M, Casanova JL, Picard C, Bustamante $\mathrm{J}$. Infectious diseases, autoimmunity and midline defect in a patient with a novel bi-allelic mutation in IL12RB1 gene. Turk J Pediatr 2016; 58: 331-336.

Clinical disease caused by weakly pathogenic mycobacterial species, which is known as Mendelian susceptibility to mycobacterial disease (MSMD), is a rare entity. IFN- $\gamma$ and IL-17 production are defective due to insufficient response to IL-2 and IL-23 in IL-12R $\beta 1$ deficiency; so this also causes tendency to intracellular microorganisms and candidal diseases. Here, we present a patient who suffers IL-12R $\beta 1$ deficiency caused by a novel bi-allelic mutation with recurrent salmonellosis, mycobacterial, fungal infections and remained asymptomatic during 13 months of follow-up after hIFN- $\gamma$ treatment. In addition she had hemolytic anemia and midline defects like cleft lip and palate which have not been reported in a patient with MSMD in the literature prior to this case report. In conclusion, diagnosis of MSMD should be kept in mind in patients with recurrent salmonellosis, mycobacterial and fungal infections especially in countries with a high consanguinity rate.

Key words: autoimmunity, IL-12R $\beta 1$ deficiency, midline defect, salmonellosis.

Mendelian susceptibility to mycobacterial disease (MSMD; MIM 209950) is a clinical syndrome that predisposes otherwise apparently healthy individuals to infections caused by weakly virulent mycobacteria, such as Bacille Calmette-Guérin (BCG) and environmental mycobacteria (EM; also known as atypical or nontuberculous mycobacteria), Salmonella and Candida ${ }^{1}$. Since 1996, MSMD-causing mutations have been identified in 9 genes $^{2}$. Seven of these genes are autosomal and encode the 2 chains of the interferon (IFN) $\gamma \gamma$ receptor (IFNGR1 and IFNGR2): the signal transducer and activator of transcription factor 1 (STAT1), the p40 subunit of interleukin (IL)-12 and IL-23 (IL12B), the $\beta 1$ chain shared by the IL-12 and IL-23 receptors (IL12RB1), the transcription factor of IFN regulatory factor 8 (IRF8), and ISG15, the IFN-stimulated gene 15 (ISG15) $^{2}$. Two genes are located in chromosome $\mathrm{X}$; encodes nuclear factor- $\mathrm{\kappa B}$ essential modulator (NEMO) and cytochrome beta chain $(C Y B B)^{3}$. The most common genetic etiology of MSMD is autosomal recessive (AR) IL-12R $\beta 1$ deficiency, first reported in $1998^{4}$. IL-12R $\beta 1$ deficiency is often, but not always, symptomatic. The clinical phenotype is very heterogenous and mycobacterial infections are 


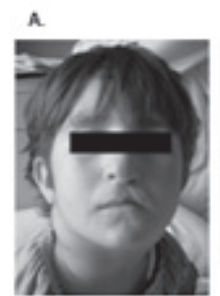

8.

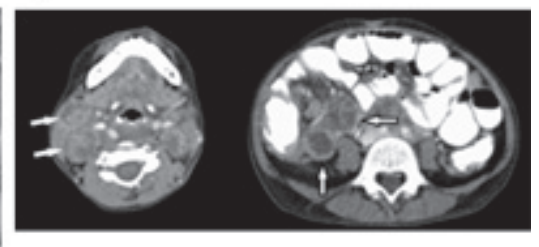

Fig. 1. Clinical manifestations. A. Facial appearance of the patient after surgery B. Appearance of paratracheal lymph nodes

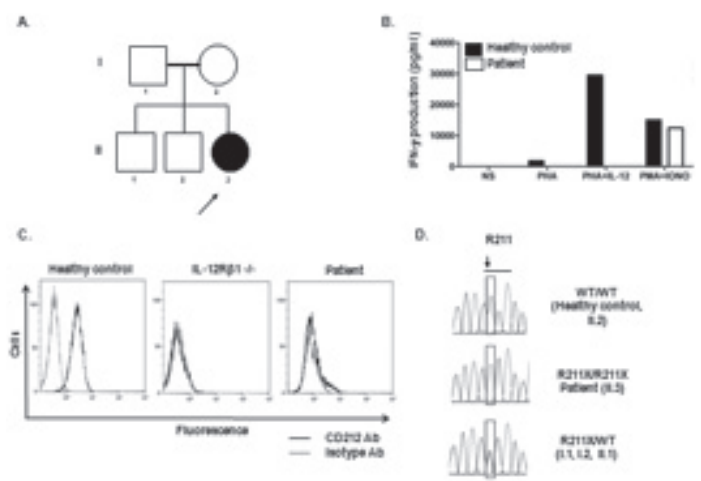

Fig. 2. IL-12R $\beta 1$ deficiency. A. Pedigree showing the index case, the siblings and both parents; each generation is designated by a roman numeral (I-II), the arrow indicates the proband. B. The patient's cells did not produce IFN- $\gamma$ after PHA+IL12 activation; however normal production of IFN- $\gamma$ is observed upon PMA-Iono activation. C. The IL-12R $\beta 1$ expression is absent on PHA-blasts from the patient. D. Sanger sequencing of IL12RB1 mutation causing R211X mutation from patient, siblings and parents
Patient

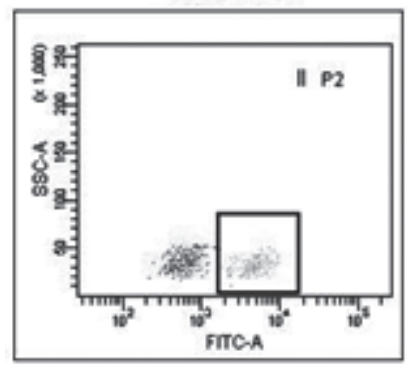

Un stimulated

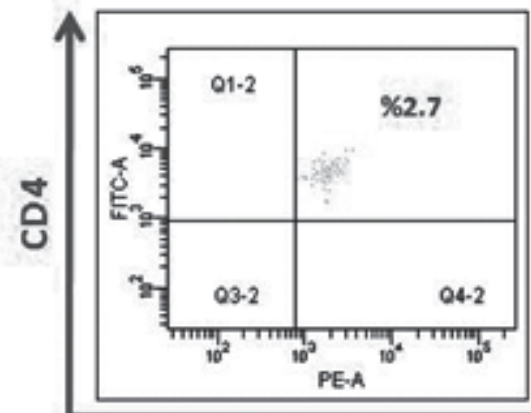

IL-17 stimulated
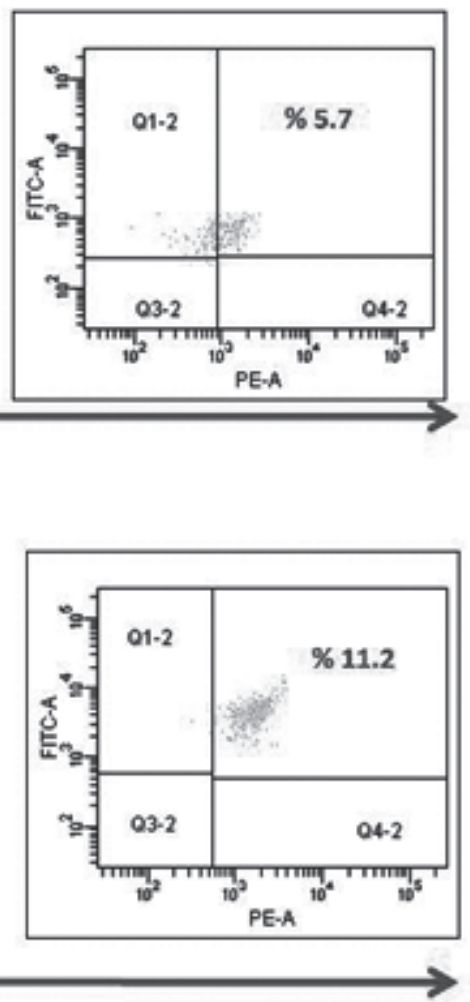

\section{IL-17}

Fig. 3. Evaluation of IL-17 pathway. IL-17 percentages and mean fluorescence intensities expressed by CD4+T cells were found to be low both before and after in vitro stimulation by PMA-Iono when compared with the healthy control. 
Table I. Evaluation of the Laboratory Parameters

\begin{tabular}{|c|c|c|c|c|c|}
\hline Laboratory parameter & Range & $\begin{array}{c}\text { Normal } \\
\text { values }\end{array}$ & Laboratory Parameter & Range & $\begin{array}{c}\text { Normal } \\
\text { values }\end{array}$ \\
\hline WBC $\left(/ \mathrm{mm}^{3}\right)$ & 13,300 & $4,000-10,000$ & $\mathrm{CD} 3+\mathrm{T}(\%)$ & 67 & $(55-79)$ \\
\hline $\mathrm{Hb}(\mathrm{g} / \mathrm{dl})$ & 6.7 & $12.1-17.2$ & $\mathrm{CD} 3+\mathrm{CD} 4+\mathrm{T}(\%)$ & 34.5 & $(26-49)$ \\
\hline $\operatorname{PLT}\left(/ \mathrm{mm}^{3}\right)$ & 375,000 & $\begin{array}{l}150,000- \\
400,000\end{array}$ & $\mathrm{CD} 3+\mathrm{CD} 8+\mathrm{T}(\%)$ & 22 & $(9-35)$ \\
\hline ANS $\left(/ \mathrm{mm}^{3}\right)$ & 7,200 & $1,500-7,300$ & CD3+CD4-CD8-T (\%) & $10-28$ & $<5 \%$ \\
\hline $\operatorname{ALS}\left(/ \mathrm{mm}^{3}\right)$ & 2,800 & $1,000-5,500$ & $\begin{array}{c}\text { CD3+CD4-CD8-TCR A/ } \\
\text { B+(\%) }\end{array}$ & 0.5 & $<2$ \\
\hline $\operatorname{AES}\left(/ \mathrm{mm}^{3}\right)$ & 790 & $200-600$ & CD3+TCR a/b (\%) & 56 & $>50 \%$ \\
\hline $\operatorname{ESR}(\mathrm{mm} / \mathrm{h})$ & 120 & $0-1$ & CD3+TCR g/d (\%) & $13-34$ & $<10 \%$ \\
\hline C-RP (mg/L) & 123 & $0-5$ & CD45 RA (\%) & 64 & $(61-87)$ \\
\hline $\operatorname{IgE}(\mathrm{IU} / \mathrm{ml})$ & $422-3,660$ & $<100$ & CD45 RO (\%) & 27.5 & $(16-38)$ \\
\hline $\operatorname{IgG}(\mathrm{mg} / \mathrm{dl})$ & $\begin{array}{l}1,500- \\
5,630\end{array}$ & $(776-1,195)$ & CD19+B (\%) & $6-19$ & $(11-31)$ \\
\hline IgM (mg/dl) & $174-398$ & $(65-146)$ & CD16+56 (\%) & 10 & $(5-28)$ \\
\hline $\operatorname{IgA}(\mathrm{mg} / \mathrm{dl})$ & $101-346$ & $(54-129)$ & HLA-DR (\%) & 31 & $(18-38)$ \\
\hline AntiHBs (mIU/ml) & 79 & $>10$ & LBT $(\%)$ & 52 & $>50$ \\
\hline $\begin{array}{l}\text { Anti-B (isohemaglutinin } \\
\text { titer) }\end{array}$ & $1 / 8$ & $<1 / 10$ & CD25 activation & Normal & - \\
\hline Direct Coombs test & +++ & Negative & Neutrophil functions & Normal & - \\
\hline Reticulocyte (\%) & 7 & $0.5-2 \%$ & ANA & $-/-/-$ & - \\
\hline $\begin{array}{l}\text { Anti-neutrophill, } \\
\text { Anti-thyroid antibodies }\end{array}$ & negative & $<0.5 \%$ & $\mathrm{RF}(\mathrm{IU} / \mathrm{mL})$ & 706 & $(0-20)$ \\
\hline Procalcitonin (ng/ml) & 0.05 & $<0.5$ & Microorganisms detected & $\begin{array}{c}\text { E. coli, } \\
\text { Candida spp., } \\
\text { Salmonella }\end{array}$ & $x^{\circ}$ \\
\hline $\begin{array}{l}\text { Complement } 3 \text { (g/L) } \\
\text { Complement } 4 \text { (g/L) }\end{array}$ & $\begin{array}{c}0.79 \\
0.160\end{array}$ & $\begin{array}{c}0.55-1.2 \\
0.15-0.42\end{array}$ & $\begin{array}{c}\text { IL-17 expression (\%) } \\
\text { (Unstimulated/stimulated) }\end{array}$ & $\begin{array}{c}\text { group A, EBV } \\
2.7 / 5.7\end{array}$ & $5.4 / 11.2$ \\
\hline
\end{tabular}

WBC: white blood cell, Hb: hemoglobin, PLT: platelet, ANS: absolute neutrophil count, ALS: absolute lymphocyte count, AES: absolute eosinophil count, ESR: eryhthrocyte sedimentation rate, C-RP: C reactive protein, LBT: lymphoblastic transformation response to PHA, CD25 activation: CD25 activation of T cells after stimulation with PHA, neutrophil functions: chemotaxis, phagocytosis and respiratory burst, ANA: anti nuclear antibody, RF: rheumatoid factor, IL-17 expression: IL-17 expression basal/after in vitro stimulation by PMA+ionomycin

the most frequent infections. It typically begins in childhood and is lethal in up to a third of patients, particularly in patients with EM disease, and its prognosis seems to improve with age ${ }^{5}$. A majority of the patients suffers also salmonellosis and a $27 \%$ of reported patients also develop fungal disease, especially due to Candida ${ }^{2,6}$. The association of IL-12RR1 deficiency with other inherited diseases (due to mutations in other genes), including $\alpha 1$ antitrypsin deficiency, ataxia-telangiectasia, neurofibromatosis, and thrombophilia has been reported; and this deficiency has also been reported to be associated with other diseases of no known genetic etiology, such as IgA deficiency ${ }^{2}$. Here, we present a case of IL-12Rß1 deficiency with fungal infection and Salmonellosis associated with other clinical manifestations, such as autoimmune hemolytic anemia and cleft lip and palate.

\section{Case Report}

Patient was born in Turkey in 2005. She was the third child of consanguineous Turkish parents. Her family history was unremarkable including two healthy brothers (Fig. 1 and 2A). She was vaccinated with BCG at age 2 months without secondary effects. She had been operated for cleft palate and lip at 4.5 month-old and 18 months old, respectively (Fig. 1A). At 18 months of age, she had been suffering from recurrent oral moniliasis and lymphadenopathies (LAPs), culture was negative for all microbes, including mycobacteria. At 5 and a half year-old, she was referred for persistent LAPs, long lasting fever and recurrent oral moniliasis. Past history 
revealed demonstration of granulomatous inflammation on pathological examination of excised left axillary LAP $(2 \times 2 \mathrm{~cm})$, detection of $E$. coli from the cultures. She received antituberculous treatment including amikacin for two months, rifampicin and isoniasid for 9 months leading to resolution of LAPs despite lack of evidence of tuberculosis. On physical examination, she was a well developed child with oral and vaginal candidiasis, cervical lymph nodes and operation scar of cleft lip and palate seeming otherwise healthy (Fig. 1A). On follow-up, multiple lymph nodes on mediastinal, paraaortic, mesenteric and cervical region and vasculitic rash on lower extremities developed (Fig. 1B). Salmonella group A was found on blood, throat and lymph node biopsy cultures. Laboratory examination revealed high eryhthrocyte sedimentation rate (ESR), white blood cells (WBC), C reactive protein (C-RP), IgE and IgG levels, and these values persisted to be high. IgM, IgA and absolute eosinophil counts ranged between normal or high levels. Neutropenia (absolute neutrophil count: 860/ $\mathrm{mm}^{3}$ ) was detected once and anti-neutrophil antibodies were negative. No lymphopenia was detected. Hemoglobin (Hgb) was between 6.7$10 \mathrm{~g} / \mathrm{dl}$ with $8 \%$ of reticulocytes and positive Coombs test $(+++)$ suggesting autoimmune hemolytic anemia diagnosis. Phagocytosis, chemotaxis and burst studies were normal. Flow cytometric analysis was normal except high double negative and CD3+TCR $\gamma / \delta$ cells. CD $3+$ CD $4-C D 8-T C R \alpha / \beta+$ cell ratio was normal $(0.5 \%)$ which was checked to exclude autoimmune lymphoproliferative syndrome. Gradually CD19+B cells decreased $(5.7 \%)$ and CD3+CD4-CD8- double negative $\mathrm{T}$ cells $(28 \%)$ and $\mathrm{CD} 3+\mathrm{TCR} \alpha / \beta+$ cells increased (34\%). Antinuclear antibody was negative for 3 times. Rheumatoid factor was $706 \mathrm{IU} / \mathrm{mL}$ (0-20). Serologies of EBV EBNA IgG, EBV EBNA IgM, EBV VCA and IgG were positive, salmonella and brucella agglutination, HSV, Parvovirus, CMV, Rubella, and Toxoplasma antibodies were negative, TBC RNA, ARB were negative on gastric lavage during a febrile episode (Table I).

Although autoimmunity was not expected as a classical component of MSMD, the IFN- $\gamma$ defect was considered to be the probable diagnosis regarding recurrent LAPs with granulomatous inflammation of unknown etiology, good response to tuberculosis treatment, Salmonella infection and non-invasive candidal infectious disease. Activation of peripheral blood mononnuclear cells (PBMCs) by phytohemagglutinin (PHA) in association with IL-12 shows an absence of production of IFN- $\gamma$ in patient's cells; in contrast, production of IFN- $\gamma$ upon Phorbol 12-myristate 13-acetate/ Ionomycin (PMA-Iono) is similar to the healthy control (Fig. 2B). In addition, T-blasts from the patient did not express IL-12R $\beta 1$ in surface (Fig. 2C). Genetical evaluation by Sanger method revealed a novel homozygous mutation (R211X) in exon 7 in the IL12RB1 gene (Fig. 2D). Both parents and one brother are heterozygous for this mutation; and a second brother is wild type (Fig. 2D). IL-17 percentages and mean fluorescence intensities expressed by CD4+T cells were found to be low both before and after in vitro stimulation by PMA-Iono when compared with the healthy control as described in patients with IL-12R $\beta 1$ deficiency (Fig. 3) ${ }^{7}$. On follow-up, she was re-hospitalized due to inguinal lymphadenitis due to Salmonella infection. Recombinant hIFN- $\gamma$ (Imukin) was given at the recommended dosage of $50 \mu \mathrm{g} / \mathrm{m}^{2}$ by subcutaneous route three times a week. The patient remained asymptomatic during 13 months of follow-up after hIFN- $\gamma$ treatment. She received prophylaxis with fluconazole.

\section{Discussion}

We describe here a patient with salmonellosis, mycobacterial and fungal infections. The patient suffers a IL-12R $\beta 1$ deficiency caused by a novel bi-allelic mutation. In addition she had hemolytic anemia and midline defects like cleft lip and palate. Up to date, these two last components has not been reported in a patient with IL-12R $\beta 1$ deficiency in the literature prior to this case report.

Chronic mucocutaneous candidiasis (CMC) is a striking feature of some immunodeficiencies. Also about $27 \%$ of IL-12p40- and IL-12R $\beta 1$ deficient patients also suffer from mild signs of CMC (but not dermatophytosis), even when not clinically ill from other infections or on antibiotic treatment ${ }^{2,4,5}$. The common defect detected in these diseases seems to be IL-17 deficiency, which causes defective cutaneous antifungal immunity ${ }^{8}$. It has been demonstrated that patients with IL-12R $\beta 1$ 
deficiency display impaired development of IL17-producing T cells, although this impairment is less pronounced than that in STAT3-deficient patients ${ }^{7}$. Also, the cells of all patients have an impaired response to IL-12 and IL-23, resulting in the impaired production of IFN- $\gamma$ and IL-175. The patient described here had oral and vaginal candidiasis since 18 month of age which were responsive to oral fluconazole treatment. She had low IL-17 levels before and after stimulation by PMA-Iono.

Drug induced autoimmune hemolytic anemia due to ciprofloxacin treatment for Salmonella infection has been reported ${ }^{9}$. Other than this, an adult patient with anti IFN- $\gamma$ antibodies was shown to have autoimmune hemolytic anemia during an episode of Salmonella infection, but the etiopathogenesis is unknown ${ }^{10}$. Probably Salmonella infection contributes in the pathogenesis, like cutaneous leukocytoclastic vasculitis described in few reports of MSMD patients with salmonellosis ${ }^{11,12,13}$. The cytokine IL-17 is also involved in the pathogenesis of several experimental autoimmune diseases ${ }^{14}$. It was indicated that mechanisms mediated by Th17 could have a role in both $\mathrm{T}$ cell- and Ab-mediated autoimmunity. The association of MSMD and autoimmune hemolytic anemia has not been reported in the literature prior to this case report.

Cleft lip and palate are the most common presenting congenital physical defect. The cause of isolated clefting is multiple involving environmental and genetic factors ${ }^{15}$. Up to date, no case report of MSMD with cleft lip or palate has been reported. Midline tongue and palate anomalies which ranged from mild forms to clefts were reported in almost half of the patients with Hyper IgE syndrome (HIES) ${ }^{16}$. These anomalies might indicate a possible developmental fusion defect in patients with HIES. Both IFN- $\gamma$ production and IL-17 production were found to be low in HIES patients ${ }^{8}$. Sharova et al. ${ }^{17}$ compared the effects of two different immunostimulators: Freund's complete adjuvant (FCA) and IFN- $\gamma$ on urethane-induced teratogenesis and they found that both FCA and IFN- $\gamma$ reduced the severity of clefting in mice. Punareewattana et al. ${ }^{18}$ previously reported that three diverse immune stimulators, CFA, GM-CSF, and IFN- $\gamma$, each significantly reduced the incidence of birth defects caused by diabetes mellitus. So, IFN- $\gamma$ deficiency could be related with midline fusion defects. Identification of IL12RB1 mutation in the patient was made by Sanger method. As the patient was born to a consanguineous family, probably another mutation located in another gene could explain this association. New technologies in genetics, such as exomesequencing or Copy Number variation (CNV) could discover the genetic cause of this defect.

In conclusion; IFN- $\gamma$ and IL-17 production are defective due to insufficient response to IL-2 and IL-23 in IL-12R $\beta 1$ deficiency; so this causes tendency to intracellular microorganisms and candidal diseases. Our case differs regarding marked oral moniliasis, lymphadenopathies, accompanied by autoimmunity and cleft lip and palate with a novel bi-allelic mutation in the IL12RB1 gene. Diagnosis of MSMD should be kept in mind in countries in which consanguinity rate is high and BCG vaccine administration is routine in early infancy. Both curative and preventive treatment of IL$12 \mathrm{R} \beta 1$ deficiency, based on prolonged courses of antibiotics, exogenous IFN- $\gamma$ treatment and, in rare cases, surgical resection of affected areas, may influence clinical outcome in these patients.

\section{Acknowledgements}

We want to thank Yelena Nemirovskaya, and Lahouari Amar for their administrative support. This research was funded in part by the National Institute of Allergy and Infectious Diseases grant number 5R01AI089970, the National Center for Research Resources and the National Center for Advancing Sciences of the National Institutes of Health grant number 8UL1TR000043, The Rockefeller University, the St. Giles Foundation, Institut National de la Santé et de la Recherche Médicale (INSERM), Paris Descartes University, Laboratoire d'Excellence Integrative Biology of Emerging Infectious Diseases (ANR-10-LABX62-IBEID) and the French National Research Agency.(ANR) under the "Investments for the future” (grant number ANR-10-IAHU-01).

\section{REFERENCES}

1. Casanova JL, Abel L. Genetic dissection of immunity to mycobacteria: the human model. Annu Rev Immunol 2002; 20: 581-620. 
2. Bustamante J, Boisson-Dupuis S, Abel L, Casanova JL. Mendelian susceptibility to mycobacterial disease: genetic, immunological, and clinical features of inborn errors of IFN- $\gamma$ immunity. Semin Immunol 2014; 26: 454-470.

3. Filipe-Santos O, Bustamante J, Haverkamp $\mathrm{MH}$, et al. X-linked susceptibility to mycobacteria is caused by mutations in NEMO impairing CD40-dependent IL-12 production. J Exp Med 2006; 203: 1745-1759.

4. Prando C, Samarina A, Bustamante J, et al. Inherited IL-12p40 deficiency: genetic, immunologic, and clinical features of 49 patients from 30 kindreds. Medicine (Baltimore) 2013; 92: 109-122.

5. de Beaucoudrey L, Samarina A, Bustamante J, Cobat A, et al. Revisiting human IL-12R $\beta 1$ deficiency: a survey of 141 patients from 30 countries. Medicine (Baltimore) 2010; 89: 381-402.

6. Zahid MF, Ali SA, Jehan F, et al. Recurrent Salmonellosis in a Child with Complete IL-12R $\beta 1$ Deficiency. J immunodefic Disord 2014; 4: 3.

7. de Beaucoudrey L, Puel A, Filipe-Santos O, et al. Mutations in STAT3 and IL12RB1 impair the development of human IL-17-producing T cells. J Exp Med 2008; 205: 1543-1550.

8. Puel A, Picard C, Cypowyj S, Lilic D, Abel L, Casanova JL. Inborn errors of mucocutaneous immunity to Candida albicans in humans: a role for IL-17 cytokines?. Curr Opin Immunol 2010; 22: 467-474.

9. MacKay AD, Mehta A. Autoimmune haemolytic anaemia associated with ciprofloxacin. Clin Lab Haematol 1995; 17: 97-98.

10. Chaisathaphol T, Jitmuang A. Disseminated Mycobacterium avium and recurrent Salmonella group $\mathrm{D}$ infection in a patient with autoantibodies to interferon-gamma. Southeast Asian J Trop Med Public Health 2013; 44: 460-467.
11. Sanal O, Turul T, De Boer T, et al. Presentation of Interleukin-12/-23 Receptor $\beta 1$ Deficiency with Various Clinical Symptoms of Salmonella Infections. J Clin Immunol 2006; 26: 1-6.

12. Filiz S, Kocacik Uygun DF, Verhard EM, et al. Cutaneous leukocytoclastic vasculitis due to Salmonella enteritidis in a child with interleukin-12 receptor beta-1 deficiency. Pediatr Dermatol 2014; 31: 236-240.

13. Kutukculer N, Genel F, Aksu G, et al. Cutaneous leukocytoclastic vasculitis in a child with interleukin-12 receptor beta-1 deficiency. J Pediatr 2006; 148: 407409.

14. Komiyama Y, Nakae S, Matsuki T, et al. IL-17 plays an important role in the development of experimental autoimmune encephalomyelitis. J Immunol 2006; 177: 566-573.

15. Gil-da-Silva-Lopes VL, Monlleo IL. Risk factors and the prevention of oral clefts. Braz Oral Res 2014; 28: $1-5$.

16. Domingo DL, Freeman AF, Davis J, et al. Novel intraoral phenotypes in hyperimmunoglobulin-E syndrome. Oral Dis 2008; 14: 73-81.

17. Sharova L, Sura P, Smith BJ, et al. Nonspecific stimulation of the maternal immune system. II. Effects on gene expression in the fetus. Teratology 2000; 62: 420-428.

18. Punareewattana K, Sharova LV, Li W, Ward DL, Holladay SD. Reduced birth defects caused by maternal immune stimulation may involve increased expression of growth promoting genes and cytokine GM-CSF in the spleen of diabetic ICR mice. Int Immunopharmacol 2003; 3: 1639-1655 